\title{
THE NUMERICAL SOLUTION BY THE METHOD OF DIRECT INTEGRALS OF DIFFERENTIATION OF EQUATIONS HAVE AN APPLICATION IN THE GAS FILTRATION THEOREM
}

\author{
Abdujabbor Abdurazakov \\ Candidate of Physics Sciences, \\ Associate Professor, \\ Ferghana Polytechnic Institute, \\ Uzbekistan, \\ Ferghana
}

\author{
Nasiba Makhmudova \\ Senior Lecturer, \\ Ferghana Polytechnic Institute, \\ Uzbekistan, \\ Ferghana
}

\author{
Nilufar Mirzamakhmudova \\ Senior Lecturer, \\ Ferghana Polytechnic Institute, \\ Uzbekistan, \\ Ferghana
}

Article DOI: https://doi.org/10.36713/epra5368

\section{ANNOTATION}

On the basis of the direct method and a combination of differential sweep, the article developed a calculated algorithm for solving gas filtration, thereby taking into account the convergence of the approximate solution to the exact one.

KEYWORDS: direct method, sweep method, differential equation, time step, convergence, approximate solution, error estimate.

\section{ANALYSIS}

The problems of non-stationary filtering are of theoretical and practical interest [1]. Consider gas filtration taking into account pressure and velocity relaxation

The problem is to find in the region

$$
\bar{\Omega}=\{0 \leq x \leq 1,0 \leq t \leq T\} \text { of a continuous function }
$$

$u(x, t)$ satisfying in the equation

$$
\frac{1}{m(x)} \frac{\partial}{\partial x}\left(k(x) \frac{\partial u}{\partial x}\right)=M(x, t, u) \frac{\partial u}{\partial t}+f(x, t, u)+\int_{0}^{t} R(t, s) d s
$$

Initial condition

$$
u(x, 0)=\varphi(x), x \in[0,1]
$$


The boundary conditions are chosen depending on the convergence of the integrals

$$
\begin{aligned}
& \int_{0}^{1} \frac{d x}{k(x)} \text { and } \int_{0}^{1} \frac{\int_{0}^{x} m(\xi) d \xi}{k(x)} d x \\
& \text { If } \int_{0}^{1} \frac{d x}{k(x)}<+\infty \text {, then }
\end{aligned}
$$

$$
\left.k(x) \frac{\partial u}{\partial x}\right|_{x=0}=\left.k(x) \frac{\partial u}{\partial x}\right|_{x=1}=0
$$

If $\int_{0}^{1} \frac{d x}{k(x)}=+\infty, \quad \int_{0}^{1} \frac{\int_{0}^{x} m(\xi) d \xi}{k(x)} d x<+\infty$, then the conditions for $x=0$ are replaced by the condition

$$
|u(x, 0)|_{x=0} \mid<+\infty
$$

Here $k(x), m(x), f(x, t, u), M(x, t, u), R(t, s)$-the given functions in the field of changing their arguments, $k(0)=0, k(x)$ and $m(x)$ moreover, are positive for $x>0, M(x, t, u) \geq m_{0}$ in the field $\{0 \leq x \leq 1,0 \leq t \leq T,|u|<+\infty\}$

We assume that all known functions in the $\bar{\Omega}$ equation are sufficiently $t=t_{j}$ smooth $t_{j}=j \tau, \quad j=1, \ldots, N, N=\left[\frac{T}{\tau}\right]$

We denote by the $u_{j}(x)$ approximate value of the desired function on the line $t=t_{j}$. We approximate the problems by the following scheme

$$
\begin{aligned}
& \frac{1}{m(x)} \frac{d}{d x}\left(k(x) \frac{d u_{j}}{d x}\right)=M\left(x, t_{j}, u_{j-1}\right) \delta_{t} u_{j}+f\left(x, t_{j}, u_{j-1}\right)+\tau \sum_{i=0}^{j-1} R_{j, i} u_{i}, \\
& j=\overline{1, n}
\end{aligned}
$$

$$
u_{0}(x)=\varphi(x)
$$

If $\int_{0}^{1} \frac{d x}{k(x)}<+\infty$, then the boundary conditions 


$$
\left.k(x) \frac{d u_{j}}{d x}\right|_{x=0}=\left.k(x) \frac{\partial u_{j}}{\partial x}\right|_{x=1}=0 \quad j=\overline{1, n}
$$

And if $\int_{0}^{1} \frac{d x}{k(x)}=+\infty, \quad \int_{0}^{1} \frac{\int_{0}^{x} m(\xi) d \xi}{k(x)} d x<+\infty$

then the conditions for $x=0$ replaced by conditions

$$
\mid u_{j}(x) \|_{x=0}<+\infty, j=\overline{1, n}
$$

Where

$$
\delta_{t}-u_{j}=\frac{u_{j}-u_{j-1}}{\tau}, j=\overline{1, N}
$$

Problem (1) - (7) is solved sequentially from layer to layer starting $j=1$, and each time there is a unique solution corresponding to the boundary value problem (1) - (2) [1].

Estimating the solutions to problem (1) - (7), we obtain

$$
\left\|u_{j}(x)\right\| \leq\left|\frac{\frac{-M\left(x, t_{j}, u_{j-1}\right)}{\tau} u_{j-1}+\tau \sum_{i=0}^{j-1} R_{j, i} u_{i}+f\left(x, t_{j}, u_{j-1}\right)}{\frac{-M\left(x, t_{j}, u_{j-1}\right)}{\tau}}\right| \leq\left(1+c_{2} T \tau\right)\|u\|_{j-1}+c_{1} \tau, \quad j=\overline{1, n}
$$

Hence

$\|u\|_{j} \leq\left(1+c_{2} T \tau\right)\|u\|_{j-1}+c_{1} \tau, \quad j=1, \ldots, N$

where

$\|u\|_{j}=\max _{1 \leq k \leq i}\left|u_{k}\right| ; \quad\|\circ\|=\max |\circ|, \quad j=1, \ldots, N$

then easy to get $\|u\|_{N} \leq\|\varphi\| e^{c_{2} T^{2}}+\frac{c_{1}}{T c_{2}}\left(e^{c_{1} T^{2}}-1\right)$

and also $\left\|u_{j}\right\| \leq\|\varphi\| e^{c_{2} T^{2}}+\frac{c_{1}}{T c_{2}}\left(e^{c_{1} T^{2}}-1\right)$ for all $j=1, \ldots, N$

Where the constants and - depend only on the given functions. The estimate is based on the maximum principle [1], [3]. 
Similarly, we prove the uniform boundedness of the following quantities.

$$
\left|\delta_{t} u_{j}\right|,\left|k(x) \frac{d u_{j}}{d x}\right|,\left|\frac{1}{m(x)} \frac{d}{d x} k(x) \frac{d u_{j}}{d x}\right|,\left|\delta_{t}\left(\delta_{t} u_{j-1}\right)\right|,\left|k(x) \frac{d \phi_{j}}{d x}\right|,\left|\frac{1}{m(x)} \frac{d}{d x} k(x) \frac{d \phi_{j}}{d x}\right|
$$

for all $j=1, \ldots, 10, \phi_{j}=\delta_{t} u_{j}$

Uniform limited functions $\left|\frac{d u_{j}}{d x}\right|,\left|\frac{d \phi_{j}}{d x}\right|$ depending on $\lim _{x \rightarrow 0} \int_{0}^{x} \frac{d x}{k(x)}$

Let $\lim _{x \rightarrow 0} \int_{0}^{x} \frac{d x}{k(x)}$ it exist and be finite.

We write analytically the linear extension formula

$$
E^{\tau}(x, t)=\frac{t-t_{j-1}}{\tau} u_{j}(x)+\frac{t_{j}-t}{\tau} u_{j-1}, j=1, \ldots, N
$$

We construct functions $u^{\tau}(x, t), u_{t}^{\tau}(x, t), k(x) u_{x}^{\tau}, \frac{1}{m(x)} \frac{\partial}{\partial x} k(x) \frac{\partial u^{\tau}}{\partial x}$ using linear extension for $t \in\left[t_{j-1} ; t_{j}\right], j=\overline{1, N}$

The resulting family depends on the way the segment is split $[0, T]$.

The estimates obtained $\Omega$ imply uniform roundedness and equidistant continuity in, a family of functions $u^{\tau}(x, t), u_{t}^{\tau}(x, t), k(x) u_{x}^{\tau}$

These families are compact in uniform convergence. Therefore, it is possible to choose a sequence $\left\{\tau_{j}\right\}$ such that $\tau_{j} \rightarrow 0$, and the sequence $\left\{u^{\tau_{j}}\right\},\left\{u_{t}^{\tau_{j}}\right\},\left\{k(x) u_{x}^{\tau_{j}}\right\}$ converges uniformly in $\Omega$ and it follows that the sequence $\left\{u^{\tau_{j}}\right\}$ converges equally in the region $\Omega_{\delta}=\{\delta \leq x \leq 1,0 \leq t \leq \tau\}$ where $0 \leq \delta \leq 1$. Due to randomness $\delta$, we conclude that, $\left\{u_{x}^{\tau_{j}}\right\}$ converges at $\tau_{j} \rightarrow 0$ at each point $\Omega_{\delta}=\{\delta \leq x \leq 1,0 \leq t \leq T\}$.

In view of the linear extension formula, we have

$$
\begin{aligned}
& \frac{1}{m(x)}\left(k(x) u_{x}^{\tau}\right)_{x}^{1}-M\left(x, t, u^{\tau}\right) u_{t}^{\tau}-f\left(x, t, u^{\tau}\right)-\int_{0}^{1} R(t, s) u^{\tau}(x, s) d s=\varepsilon(\tau) \\
& \left.k(x) u_{x}^{\tau}\right|_{x=0}=\left.k(x) u_{x}^{\tau}\right|_{x=1}=0
\end{aligned}
$$

Where $\varepsilon(\tau) \rightarrow 0$ in $\tau \rightarrow 0$.

Passing to the limit in the chosen sequence, which $u(x, t)$ satisfies $\Omega$ Eq. (1) and with condition (2), (3). 
Suppose $\lim _{x \rightarrow+0} \int_{0}^{x} \frac{d u}{k(u)}=+\infty$, then it can easily be established that $\left|u^{\tau}\left(x^{\prime \prime}, t^{\prime \prime}\right)-u^{\tau}\left(x^{\prime}, t^{\prime}\right)\right| \leq c_{1}\left|\sigma\left(x^{\prime \prime}\right)-\sigma\left(x^{\prime}\right)\right|+\mu_{0}\left(t^{\prime \prime}-t^{\prime}\right)$ where $c_{1}, \mu_{1}$-is some constant.

Here $\sigma(x)=\int_{0}^{x} \frac{\int_{0}^{\xi} m(\eta) d \eta}{k(\xi)} d \xi$, an increasing absolutely continuous function in [0,1].

Reasoning as in the proof $\int_{0}^{1} \frac{d x}{k(x)}<+\infty$, we come to the assertion that in the domain $\Omega$ there exists a solution to equation (1) satisfying the initial conditions (2) and boundary by conditions (3) - (4).

The numerical implementation of the solution of problems (5) - (6) will use the modified sweep method [1],

Direct sweep: to construct a numerical solution $\alpha_{j}(x), \beta_{j}(x)$ in the field $\{0 \leq x \leq \delta\}, \delta$ - of a sufficiently small number, by the formulas

$$
\begin{aligned}
& \alpha_{j}(x)=\frac{1}{V_{j}(x)}\left(1+\int_{0}^{x} m(\xi) \frac{M\left(\xi, t_{j}, u_{j-1}\right.}{\tau} V_{j}(\xi) d \xi\right), \\
& \beta_{j}(x)=\frac{1}{V_{j}(x)}\left(1+\int_{0}^{x}\left(m(\xi) \frac{-M\left(\xi, t_{j}, u_{j-1}\right)}{\tau} u_{j-1}+\tau \sum_{i=0}^{j-1} R_{i, j} u_{i}+f\left(\xi, t_{j}, u_{j-1}\right)\right) d \xi\right) \\
& \text { where } V_{j}(x)=1+\int_{0}^{x} \frac{M\left(\xi, t_{j}, u_{j-1}\right)}{\tau} d \xi \\
& k(h)
\end{aligned}
$$

We seek the solution of integral equations in the form of a series.

$$
\begin{aligned}
& V_{j}(x)=\sum_{j=0}^{\infty} \sigma_{j}(x), \quad j=\overline{1, N} \\
& \sum_{j=0}^{\infty} \sigma_{j}(x) \text { - the series converges uniformly. }
\end{aligned}
$$
relations

By the method of successive approximations, the terms of the series are determined by the following

$$
\sigma_{0}=1, \quad \sigma_{j}(x)=1+\int_{0}^{x} \frac{\int_{0}^{h} m(\xi) \frac{M\left(\xi, t_{j}, u_{j-1}\right)}{\tau} \sigma_{j, k-1}(\xi) d \xi}{k(h)} d h \quad j=1,2, \ldots, N
$$


$\sigma_{j}(x)$ absolute continuous and monotonically increasing function. To calculate the integrals involved in the recurrence relations, the method of singling out features proposed by Kontorovich is used

After finding $\alpha_{j}(x)$ and $\beta_{j}(x), j=\overline{1, N}$ on the interval $[\delta, 1]$ using the Runge-Kutta method, we solve the system of equations

$$
\left\{\begin{array}{l}
\alpha_{j}^{x}(x)=m(t) \frac{M\left(x, t_{j}, u_{j-1}\right)}{\tau} u_{j-1} \frac{\alpha_{j}^{2}}{k(x)} \\
\beta_{j}^{\prime}(x)=\left[\frac{M\left(x, t_{j}, u_{j-1}\right)}{\tau}+\tau \sum_{j=1}^{j_{2}} R_{j} u_{j}+f\left(x, t_{j}, u_{j-1}\right)\right] m(x)-\frac{\alpha_{j}(x) \beta_{j}(x)}{k(x)}
\end{array}\right.
$$

with initial condition

$$
\begin{aligned}
& \left.\alpha_{j}(x)\right|_{x=\delta}=\alpha_{j}(\delta) \\
& \left.\beta_{j}(x)\right|_{x=\delta}=\beta_{j}(\delta), \quad j=1, \ldots, N
\end{aligned}
$$

Reverse run:

We consider the equation in the form

$$
\frac{d u_{j}}{d x}=\frac{M\left(x, t_{j}, u_{j-1}\right) u_{j}+\left(\frac{-M\left(x, t_{j}, u_{j-1}\right)}{\tau} u_{j-1}+\tau \sum_{t=1}^{j-1} R_{i, j} u_{i}+f\left(x, t_{j}, u_{j-1}\right)\right)}{k(x)}
$$

Under the initial condition

$$
u_{j}(1)=-\frac{\beta_{j}(1)}{\alpha_{j}(1)}, \quad j=1, \ldots, N
$$

This equation has singularities for $x \rightarrow+0$

If $\lim _{x \rightarrow+0} \int_{0}^{x} m(\varsigma) \frac{\mu\left(\xi, t_{j}, u_{j-1}\right)}{k(x)} d \xi(*)$ exists, of course, it can be eliminated by calculating the limits $\lim _{x \rightarrow+0} \frac{\alpha_{j}(x)}{k(x)}$ and $\lim _{x \rightarrow+0} \frac{\beta_{j}(x)}{k(x)}$ of $j=1, \ldots, N$. Eliminating these features, we find a solution according to the Runge-Kutta method for $j=1, \ldots, N$

If it does not exist, then we first construct the solution of the equation in the region $\{\delta \leq x \leq 1\}$, according to the Runge-Kutta method

Then using the built. 


$$
U_{j}(x)=\left(\frac{u_{j}(\delta)}{V_{j}(\delta)}-\int_{x}^{\delta} \frac{\left(\frac{-M\left(x, t_{j}, u_{j-1}\right)}{\tau} u_{j-1}+\tau \sum_{t=1}^{j-1} R_{i, j} u_{i}+f\left(x, t_{j}, u_{j-1}\right)\right.}{k(\xi) V_{j}(\xi)} d \xi\right) V_{j}(x), j=1, \ldots, N
$$

We find $u_{j}(x)$ in the area $[0, \delta], j=1, \ldots, N$

We propose one of the possible methods for the numerical solution of problem [1] and [6].

Note: An approximate solution constructed by the method of lines converges to an exact solution with a speed $0(\tau)$ where is a time step $\tau$

\section{LITERATURE}

1. Abdurazakov A. Sxodimost metoda pryamyx dlya integer-differentialnyx uravneniy.V sb. ”Vychislitelnaya matematika I matematicheskaya statistika",issue 8,1979, MGPX

2. Abdurazakov A, Makhmudova N, Mirzamakhmudova N. Solutions of a multi-point boundary value problem of gas filtration in multilayer layers with relaxation taken into account. Universum: technical sciences: electronic scientific journal 2019 №11 (68) p5-9

3. Bobkov V.V. Liskovets O.A. Point estimates in the Rothe method. Differential Equations 2, 1966, No. 5 p 140-640 